\title{
Association of Plasma Neurofilament Light With Neurodegeneration in Patients With Alzheimer Disease
}

Niklas Mattsson, MD, PhD; Ulf Andreasson, PhD; Henrik Zetterberg, MD, PhD; Kaj Blennow, MD, PhD; for the Alzheimer's Disease Neuroimaging Initiative

IMPORTANCE Existing cerebrospinal fluid (CSF) or imaging (tau positron emission tomography) biomarkers for Alzheimer disease (AD) are invasive or expensive. Biomarkers based on standard blood test results would be useful in research, drug development, and clinical practice. Plasma neurofilament light (NFL) has recently been proposed as a blood-based biomarker for neurodegeneration in dementias.

OBJECTIVE To test whether plasma NFL concentrations are increased in AD and associated with cognitive decline, other AD biomarkers, and imaging evidence of neurodegeneration.

DESIGN, SETTING, AND PARTICIPANTS In this prospective case-control study, an ultrasensitive assay was used to measure plasma NFL concentration in 193 cognitively healthy controls, 197 patients with mild cognitive impairment $(\mathrm{MCl})$, and 180 patients with $A D$ dementia from the Alzheimer's Disease Neuroimaging Initiative. The study dates were September 7, 2005, to February 13, 2012. The plasma NFL analysis was performed in September 2016.

MAIN OUTCOMES AND MEASURES Associations were tested between plasma NFL and diagnosis, $A \beta$ pathologic features, CSF biomarkers of neuronal injury, cognition, brain structure, and metabolism.

RESULTS Among 193 cognitively healthy controls, 197 patients with mild cognitive impairment, and 180 patients with AD with dementia, plasma NFL correlated with CSF NFL (Spearman $\rho=0.59, P<.001$ ). Plasma NFL was increased in patients with $\mathrm{MCl}$ (mean, 42.8 $\mathrm{ng} / \mathrm{L}$ ) and patients with $\mathrm{AD}$ dementia (mean, $51.0 \mathrm{ng} / \mathrm{L}$ ) compared with controls (mean, 34.7 $\mathrm{ng} / \mathrm{L})(P<.001)$ and had high diagnostic accuracy for patients with AD with dementia vs controls (area under the receiver operating characteristic curve, 0.87 , which is comparable to established CSF biomarkers). Plasma NFL was particularly high in patients with $\mathrm{MCl}$ and patients with $A D$ dementia with $A \beta$ pathologic features. High plasma NFL correlated with poor cognition and AD-related atrophy (at baseline and longitudinally) and with brain hypometabolism (longitudinally).

CONCLUSIONS AND RELEVANCE Plasma NFL is associated with AD diagnosis and with cognitive, biochemical, and imaging hallmarks of the disease. This finding implies a potential usefulness for plasma NFL as a noninvasive biomarker in AD.
JAMA Neurol. 2017;74(5):557-566. doi:10.1001/jamaneurol.2016.6117 Published online March 27, 2017.
E

Editorial page 510

Supplemental content
Author Affiliations: Author affiliations are listed at the end of this article.

Group Information: A list of the Alzheimer's Disease Neuroimaging Initiative investigators is given at the end of this article.

Corresponding Author: Niklas Mattsson, MD, PhD, Clinical Memory Research Unit, Department of Clinical Sciences, Faculty of Medicine, Lund University, Simrisbanvägen 14, SE-21224 Malmö, Sweden (niklas .mattsson@med.lu.se). 
A lzheimer disease (AD) is a neurodegenerative disease that is characterized by brain accumulation of $\beta$-amyloid $(A \beta)$ and tau, progressive atrophy, and cognitive decline. Biomarkers that capture biological processes in $\mathrm{AD}$ are increasingly used to support the diagnosis of $\mathrm{AD}$ in research, drug development, and clinical practice. ${ }^{1}$ The most wellestablished AD biomarkers include structural magnetic resonance imaging (MRI); cerebrospinal fluid (CSF) biomarkers of $\mathrm{A} \beta$, tau, and neuronal injury; and positron emission tomographic imaging of $A \beta$, tau, and brain metabolism..$^{2-4}$ Use of these biomarkers is hampered by a high degree of invasiveness, high costs, or limited availability. ${ }^{5}$ Blood-based biomarkers for AD may allow for efficient monitoring of disease processes in $\mathrm{AD}$ and could be used as a screening tool in primary care. One potential blood-based biomarker for $\mathrm{AD}$ is the neuronal injury marker neurofilament light (NFL) ${ }^{6}$ because patients with $\mathrm{AD}$ have increased CSF concentrations of NFL. ${ }^{7}$ Results from some studies ${ }^{8,9}$ suggest that patients with AD have increased plasma NFL concentrations. However, those studies were performed using standard immunoassay techniques with suboptimal analytical sensitivity to accurately quantify low abundant brain-specific proteins in blood samples..$^{10}$ For this reason, our group has recently transferred the CSF NFL assay to an ultrasensitive single-molecule array (Simoa; Quanterix Corporation) platform, which provides an analytical sensitivity of $0.6 \mathrm{pg} / \mathrm{mL}$ compared with $78.0 \mathrm{pg} / \mathrm{mL}$ for the corresponding enzyme-linked immunosorbent assay (ELISA). ${ }^{10}$ Plasma NFL concentrations can be measured in all samples using the ultrasensitive single-molecule array and correlate closely with the corresponding CSF concentrations. ${ }^{11,12}$ Herein, we test this novel plasma NFL assay in patients with AD for the first time, to our knowledge. We studied cognitively healthy control individuals, patients with mild cognitive impairment (MCI) (MCI group), and patients with AD dementia (AD group) in a large prospective study. We tested the hypotheses that the plasma NFL concentration is increased in $\mathrm{AD}$ and that it correlates with impaired cognition, neuroimaging abnormalities, and CSF biomarkers of $\mathrm{AD}$ pathologic features.

\section{Methods}

\section{ADNI Study Design}

Data were obtained from the Alzheimer's Disease Neuroimaging Initiative (ADNI) database (http://adni.loni.usc.edu). The ADNI was launched in 2003 as a public-private partnership, led by principal investigator Michael W. Weiner, MD (the most recent information on the ADNI is available at http://www .adni-info.org). The ADNI participants have been recruited from more than 50 sites across the United States and Canada. For the present study, we used data accessed from the Laboratory of Neuro Imaging (University of Southern California) ADNI database on October 6, 2016. The study data and samples were collected from September 7, 2005, to February 13, 2012. Regional ethical committees of all participating institutions approved the ADNI. All study participants provided written informed consent.

\section{Key Points}

Question What is the importance of plasma neurofilament light in Alzheimer disease?

Findings In this case-control study of 193 cognitively healthy controls, 197 patients with mild cognitive impairment, and 180 patients with Alzheimer disease dementia, plasma neurofilament light was associated with Alzheimer disease and correlated with future progression of cognitive decline, brain atrophy, and brain hypometabolism.

Meaning Plasma neurofilament light may be a promising noninvasive biomarker for Alzheimer disease.

\section{ADNI Participants}

Our ADNI cohort consisted of all cognitively healthy controls, patients with MCI, and patients with AD dementia with available baseline plasma NFL samples from the ADNI-1. Inclusion and exclusion criteria were described in detail previously. ${ }^{13}$ Briefly, all ADNI-1 participants were aged 55 to 90 years, had completed at least 6 years of education, were fluent in Spanish or English, and had no substantial neurological disease other than AD. Controls had Mini-Mental State Examination (MMSE) scores of 24 or higher, where lower scores indicate more impairment and higher scores less impairment (range, 0-30), and a Clinical Dementia Rating (CDR) score of 0, where lower scores indicate less impairment and higher scores more impairment (range, 0-3). Patients with MCI had MMSE scores of 24 or higher, objective memory loss tested by delayed recall of the Wechsler Memory Scale (WMS) logical memory II ( $>1$ SD below the normal mean), a CDR score of 0.5 , preserved activities of daily living, and absence of dementia. Patients with AD dementia fulfilled the National Institute of Neurological Communicative Disorders and Stroke-Alzheimer Disease and Related Disorders Association criteria for probable $\mathrm{AD},{ }^{14}$ had MMSE scores of 20 to 26 , and had CDR scores of 0.5 to 1.0.

\section{Plasma NFL}

Plasma NFL concentrations were measured using an NFL kit (NF-light; UmanDiagnostics), transferred onto the ultrasensitive single-molecule array platform using a home brew kit (Simoa Homebrew Assay Development Kit; Quanterix Corporation), as previously described. ${ }^{15}$ In the 14 analytical runs needed to complete the study, the relative error of the backcalculated concentrations was below $20 \%$ for all calibrators, run in triplicate, resulting in lower limits of quantifications of $2.2 \mathrm{ng} / \mathrm{L}$ and upper limits of quantification of $1620 \mathrm{ng} / \mathrm{L}$. All samples measured within the range spanned by the limits of quantifications, and for the low-concentration quality control sample (14 ng/L), the intra-assay coefficient of variation was $11.0 \%$ and the interassay coefficient of variation was $11.1 \%$. For the high-concentration quality control sample (137 ng/L), the corresponding coefficients of variation were $8.8 \%$ and $9.6 \%$, respectively. The measurements were performed in September 2016 by a board-certified laboratory technician using a single batch of reagents. 


\begin{tabular}{|c|c|c|c|c|}
\hline Variable & $\begin{array}{l}\text { Controls } \\
(n=193)\end{array}$ & $\begin{array}{l}\mathrm{MCl} \\
(\mathrm{n}=197)\end{array}$ & $\begin{array}{l}\text { AD Dementia } \\
(\mathrm{n}=180)\end{array}$ & $P$ Value \\
\hline Age, mean (SD), y & $75.9(4.9)$ & $74.7(7.5)$ & $75.3(7.3)$ & .58 \\
\hline Female, No. (\%) & $87(45.1)$ & $65(33.0)$ & $86(47.8)$ & .007 \\
\hline Educational level, mean (SD), y & $16.0(2.9)$ & $15.8(3.0)$ & $14.7(3.1)$ & $<.001$ \\
\hline APOE $\varepsilon 4$ genotype carriers, No. (\%) & $50(25.9)$ & $103(52.3)$ & $123(68.3)$ & $<.001$ \\
\hline Plasma NFL, mean (SD), ng/L & $34.7(21.4)$ & $42.8(29.0)$ & $51.0(26.9)$ & $<.001$ \\
\hline MMSE score, mean (SD) & $29.1(1.0)$ & $26.9(1.8)$ & $23.2(2.1)$ & $<.001$ \\
\hline CSF Aß42, mean (SD), ng/L & $207(52)$ & $165(52)$ & $134(23)$ & $<.001$ \\
\hline$A \beta+$, No./total No. $(\%)$ & $41 / 112(36.6)$ & $138 / 189(73.0)$ & $90 / 90(100)$ & $<.001$ \\
\hline CSF t-tau, mean (SD), ng/L & $68(29)$ & $102(60)$ & $126(56)$ & $<.001$ \\
\hline CSF p-tau, mean (SD), ng/L & $25(15)$ & $36(19)$ & $44(20)$ & $<.001$ \\
\hline
\end{tabular}
Abbreviations: $A \beta, \beta$-amyloid; $A D$, Alzheimer disease dementia; CSF, cerebrospinal fluid; $\mathrm{MCl}$, mild cognitive impairment; MMSE, Mini-Mental State Examination; NFL, neurofilament light; $\mathrm{p}$-tau, phosphorylated tau; t-tau, total tau.
${ }^{a} P$ values are from the Kruskal-Wallis test or Fisher exact test. $\beta$-Amyloid positivity was defined as CSF A 342 less than $192 \mathrm{ng} / \mathrm{L}$.

\section{CSF Measurements}

Cerebrospinal fluid was sampled by lumbar puncture from a subset of the participants, with CSF A $\beta 42$, CSF total tau (t-tau), and CSF phosphorylated tau (p-tau) measured using a multiplex platform (xMAP; Luminex Corporation) with a kit (INNO-BIA AlzBio3; Fujirebio Europe). Participants were classified as $A \beta$ positive or $A \beta$ negative using a previously established cutoff (CSF A $\beta 42<192 \mathrm{ng} / \mathrm{L}) .{ }^{16}$ We excluded 8 patients with $\mathrm{AD}$ dementia who were $\mathrm{A} \beta$ negative and therefore likely to be misdiagnosed. The CSF NFL concentrations were measured using a commercial ELISA (NF-light; UmanDiagnostics) and have been reported previoushy. ${ }^{7}$ In total, we included CSF data from 112 controls, 189 patients with MCI, and 90 patients with $\mathrm{AD}$ dementia.

\section{Cognition}

Cognition was assessed by MMSE, Alzheimer Disease Assessment Scale-cognitive subscale (ADAS-Cog 11), delayed recall of the WMS logical memory II, Trail-Making test part B (TMTB), and Wechsler Adult Intelligence Scale-Revised (WAIS-R) digit symbol substitution test. All tests were administered at baseline and at 6, 12, 18, 24, 36, and 48 months, except for delayed recall of the WMS logical memory II, which was not assessed at 18 months.

\section{Neuroimaging}

Structural brain images were acquired using 1.5-T MRI imaging systems with T1-weighted MRI scans using a sagittal volumetric magnetization-prepared rapid acquisition gradient echo sequence (at baseline and at 6, 12, 18, 24, 36, and 48 months). A software program (FreeSurfer; https://surfer.nmr.mgh .harvard.edu/) was used for quantification of cortical thickness and subcortical volumes. ${ }^{17}$ We used volumetric data for hippocampal volume and lateral ventricles (averaged between right and left sides). We used the mean cortical thickness for a set of regions defined a priori based on work by Jack et $\mathrm{al}^{18}$ to represent $\mathrm{AD}$ cortex (including entorhinal, inferior temporal, middle temporal, and fusiform cortex).

White matter hyperintensities (WMHs) were quantified at baseline and at $6,12,18,24,36$, and 48 months using a fully automated protocol. ${ }^{19}$ Positron emission tomography with $18 \mathrm{~F}$ fluorodeoxyglucose image data were acquired at baseline and at $6,12,18,24,36$, and 48 months. ${ }^{20}$ We created mean counts of the lateral and medial frontal, anterior, and posterior cingulate regions, as well as lateral parietal and lateral temporal regions.

\section{Statistical Analysis}

We tested associations between plasma NFL and demographic factors using the Kruskal-Wallis test and Spearman rank correlation. We tested associations between biochemical markers and between plasma NFL and diagnosis using linear regression models. We calculated diagnostic accuracies using area under the receiver operating characteristic curve (AUROC) analysis with 10 -fold cross-validated logistic regression models. We tested associations between plasma NFL concentrations and longitudinal cognition, brain structure, and brain metabolism using linear mixed-effects models. These models had random intercepts and slopes for time and an unstructured covariance matrix for the random effects and included the interaction between (continuous) time and plasma NFL as predictor. All outcome variables in linear mixed-effects models were standardized to $z$ scores to facilitate comparisons between modalities. Therefore, $\beta$ coefficients refer to standardized effects ( $\beta=1 \mathrm{implies}$ that an increase of $1 \mathrm{ng} / \mathrm{L}$ in plasma NFL was associated with a 1-SD increase in the dependent variable).

All tests were 2-sided. Statistical significance was set at $P<$.05. All regression analyses were corrected for age, sex, educational level, diagnosis, and $A P O E$ \&4 genotype, as well as intracranial volume for hippocampus and ventricles. All statistical analyses were performed using a software program (R, version 3.2.3; The R Foundation).

\section{Results}

Table 1 lists demographics for the study population. In the whole cohort, plasma NFL correlated with age (Spearman $\rho=0.35, P<.001$ ) but not with sex (median, $36.2 \mathrm{ng} / \mathrm{L}$ for men vs $37.4 \mathrm{ng} / \mathrm{L}$ for women; $P=.98$ ), educational level ( $\rho=-0.03, P=.52$ ), or APOE $\varepsilon 4$ genotype (37.7 $\mathrm{ng} / \mathrm{L}$ in carriers vs $35.6 \mathrm{ng} / \mathrm{L}$ in noncarriers, $P=.19$ ). These results were similar within diagnostic groups, except that plasma NFL concentrations were higher in APOE \&4 carriers in the MCI group (35.9 ng/L in carriers vs $39.3 \mathrm{ng} / \mathrm{L}$ in noncarriers, 
$P=.049)$ and in the AD dementia group (41.6 ng/L in carriers vs $51.5 \mathrm{ng} / \mathrm{L}$ in noncarriers, $P=.03$ ).

Plasma NFL and Other Biochemical Markers

in CSF and Plasma

Plasma NFL correlated with high CSF NFL (Spearman $\rho=0.59$, $P<.001$ ) (Figure 1) and with low CSF A 442 , high CSF t-tau, high CSF p-tau, and high plasma tau (Table 2). The strongest correlations were seen with CSF NFL, which were also present in all diagnostic groups. Plasma NFL also correlated with CSF $\mathrm{A} \beta 42$ and CSF t-tau in the MCI group, as well as with plasma tau concentrations in all diagnostic groups.

Plasma NFL in Different Diagnostic Groups

Plasma NFL concentrations were higher in the AD group compared with controls and the MCI group, as well as in the MCI group compared with controls (Figure 2A). Plasma NFL differentiated between the $\mathrm{AD}$ dementia group and controls, with an AUROC of 0.87 (Figure 2B). By comparison, the AUROCs were 0.87 to 0.90 for CSF NFL, CSF A $\beta 42$, CSF t-tau, and CSF p-tau and 0.78 for plasma tau. These AUROCs were corrected for age, sex, educational level, and APOE $\varepsilon 4$ geno-

Figure 1. Plasma Neurofilament Light (NFL) and Cerebrospinal Fluid (CSF) NFL

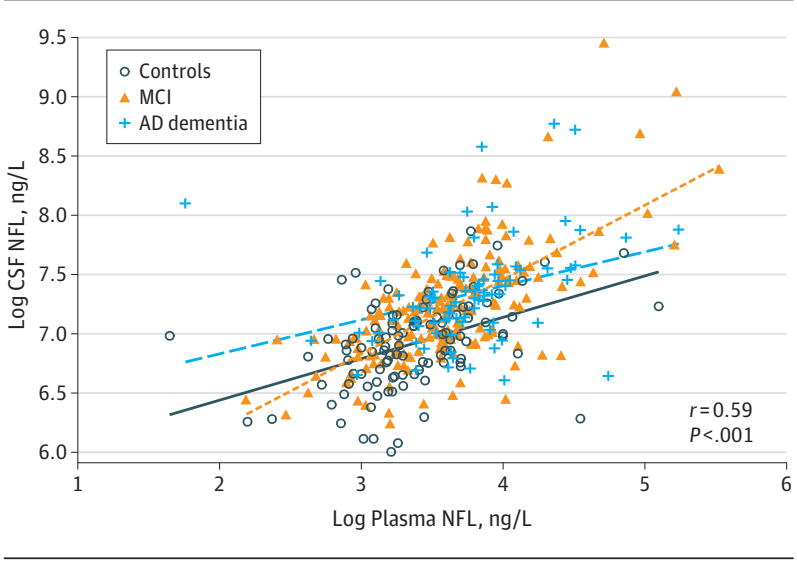

Fit lines are shown for individual diagnostic groups. The Spearman $\rho$ and $P$ values are for Spearman rank correlation in the whole cohort. Table 2 lists correlation data adjusted for covariates. AD indicates Alzheimer disease; $\mathrm{MCl}$, mild cognitive impairment. type. When only correcting for age, sex, and educational level, the AUROCs were reduced to 0.79 for plasma NFL, 0.81 for CSF NFL, 0.85 for CSF t-tau, 0.81 for CSF p-tau, and 0.64 for plasma tau.

\section{Plasma NFL and A $\beta$ Pathologic Features}

We compared plasma NFL between $A \beta$-negative controls, $A \beta$ positive controls, $A \beta$-negative patients with MCI, $A \beta$-positive patients with $\mathrm{MCI}$, and (A $\beta$-positive) patients with $\mathrm{AD}$ dementia (Figure $2 \mathrm{C}$ ). The $\mathrm{AD}$ dementia group had higher plasma NFL than $\mathrm{A} \beta$-negative controls (mean, 48.8 vs $33.9 \mathrm{ng} / \mathrm{L} ; P<.001$ ), A $\beta$-positive controls (mean, $30.9 \mathrm{ng} / \mathrm{L} ; P<.001$ ), A $\beta$-negative MCI (mean, $38.1 \mathrm{ng} / \mathrm{L} ; P<.001$ ), and A $\beta$-positive MCI (mean, $44.5 \mathrm{ng} / \mathrm{L} ; P=.05)$. There were no statistically significant differences between $A \beta$-negative and $A \beta$-positive controls and $A \beta$ negative patients with MCI.

\section{Plasma NFL and Progressive vs Stable MCI}

Among the MCI group, 109 converted to AD dementia during follow-up, and 65 remained stable after at least 2 years' follow-up. Twenty-three patients in the MCI group did not convert to $\mathrm{AD}$ dementia during follow-up but were observed for less than 2 years and were not included in the stable group. There was no difference in plasma NFL between $\mathrm{A} \beta$-positive patients with progressive $\mathrm{MCI}$ and $\mathrm{A} \beta$-positive patients with stable MCI, but both of these groups had higher plasma NFL than A $\beta$-negative patients with progressive MCI and A $\beta$-negative patients with stable MCI (Figure 2D).

\section{Plasma NFL and Cognition and Neuroimaging}

Associations between plasma NFL and longitudinal cognitive and imaging measures are shown in Figure 3 (coefficients and $P$ values are listed in the eTable in the Supplement). At baseline, high plasma NFL levels were associated with worse MMSE, ADAS-COG 11, and TMT-B scores and with larger ventricular volume, smaller hippocampal volume, and thinner cortices in the AD cortex region. Over time, high plasma NFL levels were associated with an accelerated decline in all measures, except for WMHs. The strongest influences were seen in MMSE $(\beta=-0.073, P<.001$ baseline and $\beta=-0.116, P<.001$ longitudinally) and ADAS-COG11 scores $(\beta=0.101, P<.001$ baseline and $\beta=0.106, P<.001$ longitudinally) for the cognitive measures and in $\mathrm{AD}$

Table 2. Correlations Between Plasma NFL and Other Biochemical Markers ${ }^{\mathrm{a}}$

\begin{tabular}{|c|c|c|c|c|c|c|c|c|}
\hline \multirow[t]{2}{*}{ Biomarker } & \multicolumn{2}{|c|}{ All Participants } & \multicolumn{2}{|l|}{ Controls } & \multicolumn{2}{|l|}{$\mathrm{MCl}$} & \multicolumn{2}{|l|}{ AD Dementia } \\
\hline & $\beta$ Coefficient & $P$ Value & $\beta$ Coefficient & $P$ Value & $\beta$ Coefficient & $P$ Value & $\beta$ Coefficient & $P$ Value \\
\hline CSF NFL & 0.480 & $<.001$ & 0.371 & $<.001$ & 0.615 & $<.001$ & 0.242 & .046 \\
\hline CSF Aß42 & -0.144 & .01 & 0.002 & .99 & -0.243 & $<.001$ & -0.017 & .87 \\
\hline CSF t-tau & 0.125 & .01 & 0.123 & .21 & 0.170 & .01 & 0.033 & .76 \\
\hline CSF p-tau & 0.105 & .03 & 0.068 & .49 & 0.113 & .10 & 0.112 & .31 \\
\hline Plasma tau & 0.178 & $<.001$ & 0.238 & $<.001$ & 0.138 & .03 & 0.188 & .008 \\
\hline
\end{tabular}

Abbreviations: AD, Alzheimer disease; CSF, cerebrospinal fluid; $\mathrm{MCl}$, mild cognitive impairment; NFL, neurofilament light; p-tau, phosphorylated tau; t-tau, total tau.

${ }^{\text {a }}$ Data are $\beta$ coefficients (with $P$ values) from linear regression models for

correlations between plasma NFL and other biomarkers (all standardized to $z$ scores), adjusted for age, sex, educational level, APOE $\varepsilon 4$ genotype, and diagnosis. Models were tested in the whole cohort and in individual diagnostic groups. 
A Plasma NFL by diagnosis

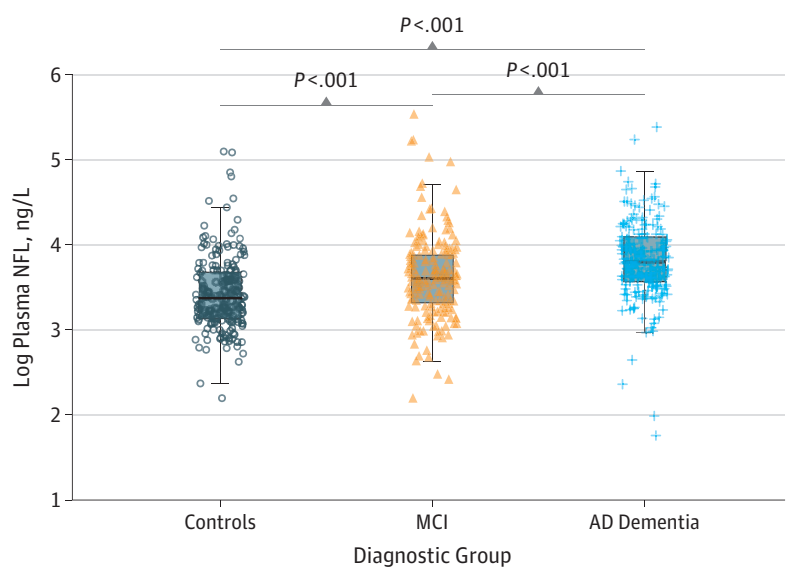

C Plasma NFL by diagnosis and AB

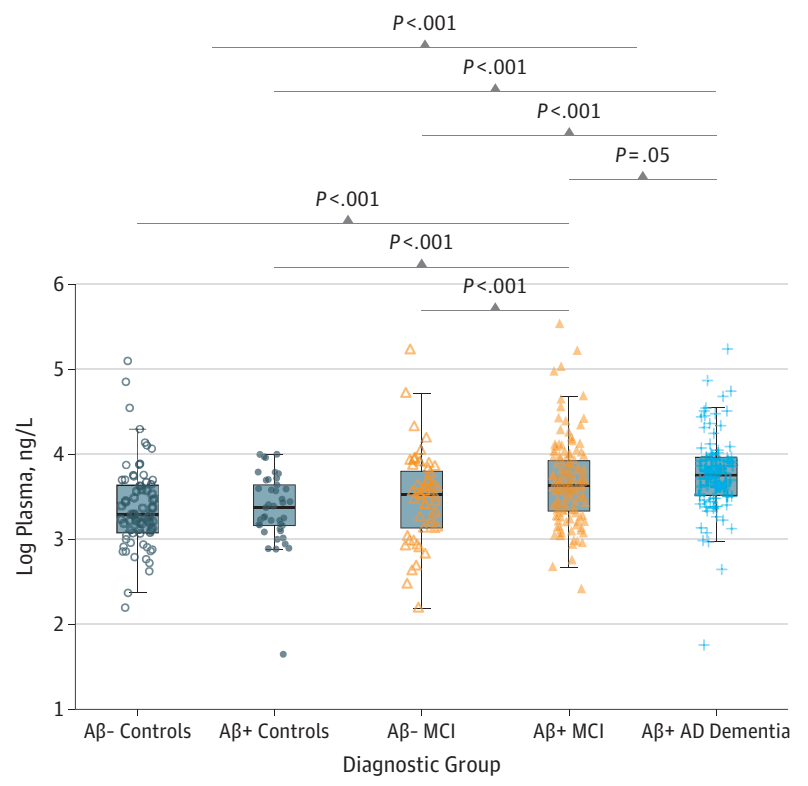

A, Plasma NFL in controls, patients with mild cognitive impairment $(\mathrm{MCl})$, and patients with Alzheimer disease (AD) dementia. B, Area under the receiver operating characteristic curve (AUROC) analyses for plasma NFL and other biomarkers to differentiate between the AD dementia group and controls. CSF indicates cerebrospinal fluid. C, Plasma NFL in controls, patients with $\mathrm{MCl}$, and patients with $A D$ dementia, stratified by occurrence of $A \beta$ positivity (CSF $\mathrm{A} \beta 42<192 \mathrm{ng} / \mathrm{L}$ ). D, Plasma NFL in patients with stable MCI (SMCI) (no
B AUROC in AD dementia vs controls

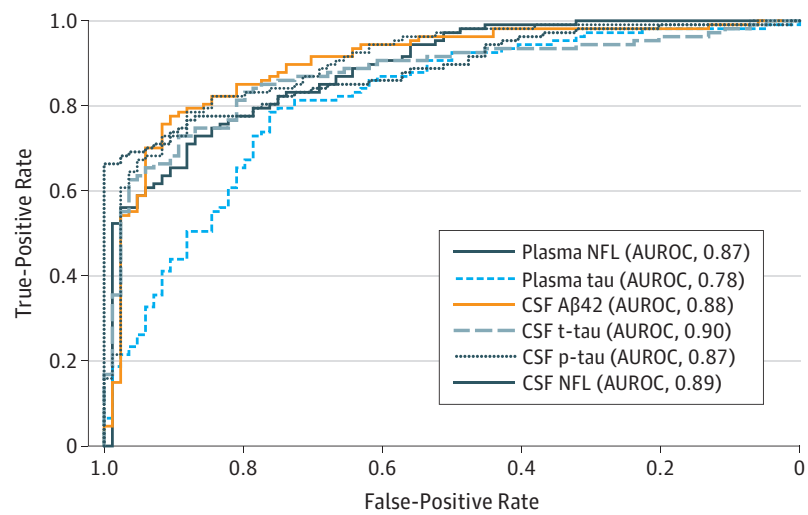

D Plasma NFL by $\mathrm{SMCl}$ or $\mathrm{PMCl}$ and $\mathrm{A} \beta$

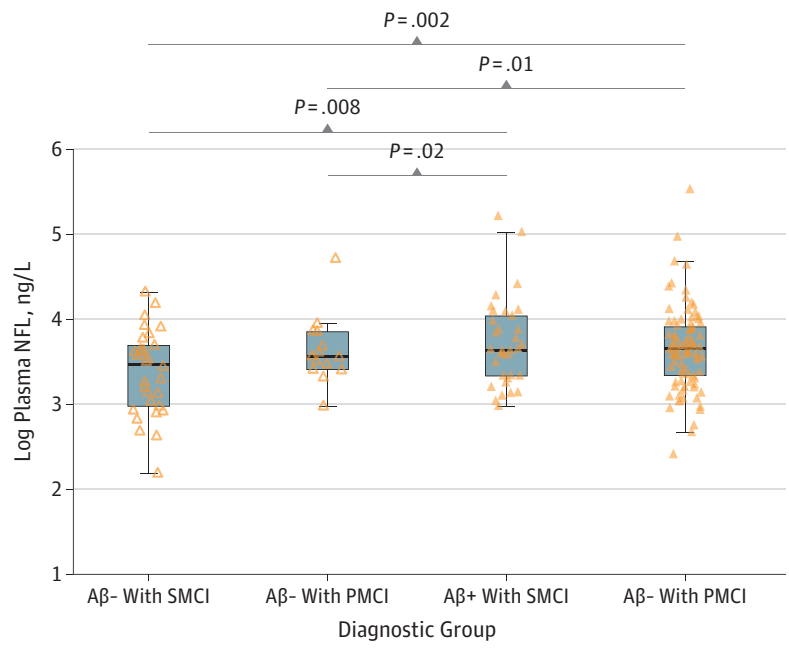

progression to dementia during $\geq 2$ years' follow-up) and patients with progressive $\mathrm{MCl}(\mathrm{PMCl}$ ) (conversion to dementia), with or without $\mathrm{A} \beta$ positivity. The models were adjusted for age, sex, educational level, and APOE \&4 genotype. Age retained an independent statistically significant association with higher plasma NFL ( $\beta=0.025, P<.001$ from the models shown in $A$ ). $A B$ indicates $A B$-negative; $A B+, A B$-positive. cortex $(\beta=-0.162, P<.001$ baseline and $\beta=-0.049$, $P<.001$ longitudinally) for the imaging measures (details are provided in the eTable in the Supplement).

We also tested whether the influence of plasma NFL differed between diagnostic groups. Statistically significant interactions were found at baseline for the MCI group and MMSE score $(\beta=-0.107, P=.008)$, ADAS-COG 11 score ( $\beta=0.150, P=.003)$, delayed recall of the WMS logical memory II $(\beta=-0.167, P<.001)$, TMT-B score $(\beta=0.283$, $P<.001)$, WAIS-R digit symbol substitution test score $(\beta=-0.253, P=.002)$, and $\mathrm{AD}$ cortex $(\beta=-0.282, P<.001)$, as well as for the AD group and MMSE score $(\beta=-0.133$, $P=.002)$, ADAS-COG 11 score $(\beta=0.171, P=.001)$, delayed recall of the WMS logical memory II ( $\beta=-0.117, P=.01)$, and AD cortex $(\beta=-0.178, P=.04)$. These results indicate that plasma NFL was more strongly correlated with the outcomes in the MCI group and the AD dementia group than in controls at baseline. Longitudinally, the only statistically significant interaction was for the MCI group and MMSE score $(\beta=-0.107, P=.003)$, demonstrating that plasma NFL 

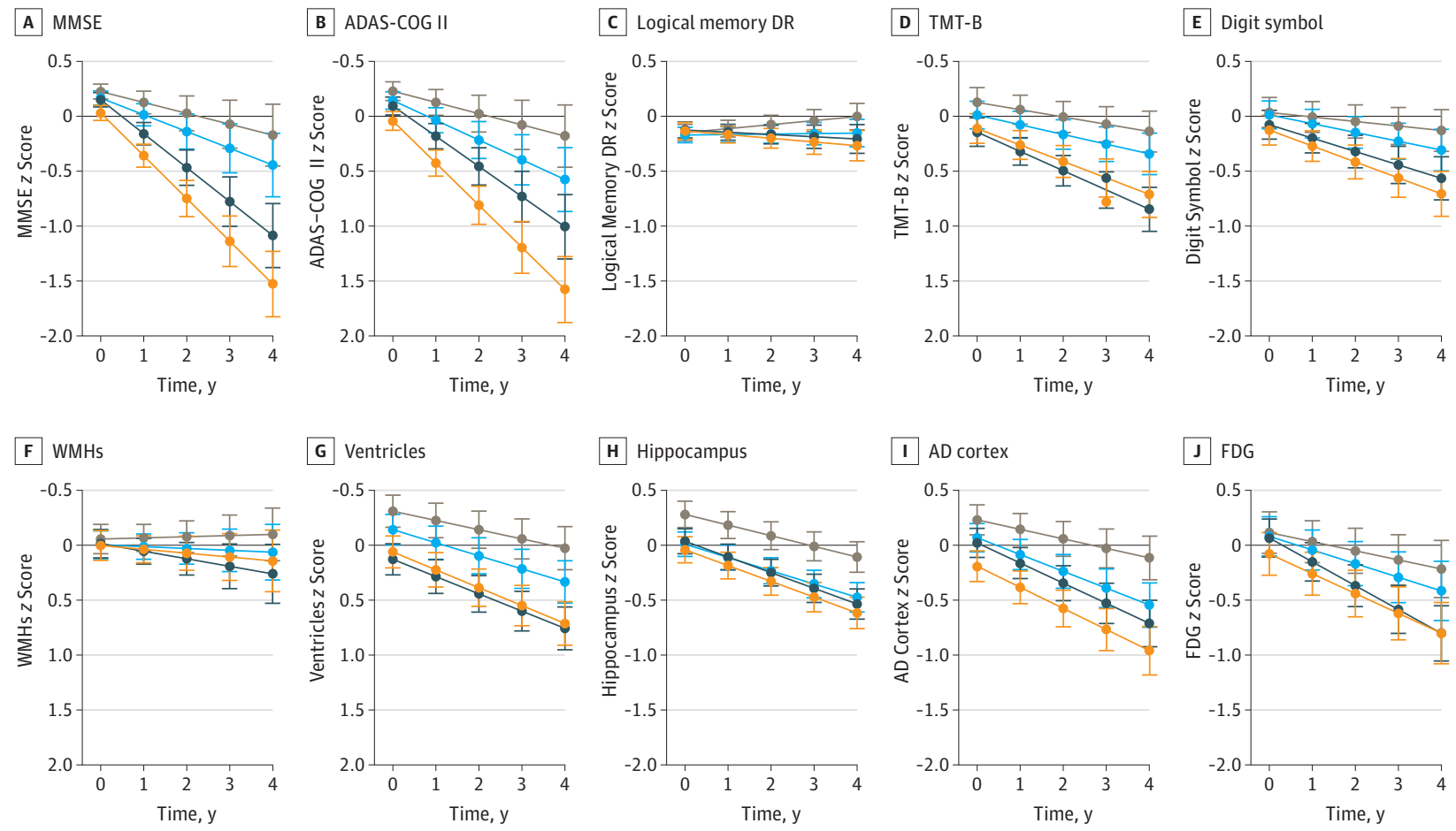

Data from linear mixed-effects models adjusted for age, sex, educational level, $A P O E \varepsilon 4$ genotype, and diagnosis, as well as intracranial volume for the neurofilament light quartiles for hippocampal volume and ventricular volume. ADAS-COG 11 indicates Alzheimer Disease Assessment Scale-cognitive subscale AD cortex, Alzheimer disease cortex; Digit symbol, Wechsler Adult Intelligence

Scale-Revised digit symbol substitution test; FDG, 18F-fluorodeoxyglucose; Logical memory DR, delayed recall of the Wechsler Memory Scale logical memory II; MMSE, Mini-Mental State Examination; Q, quartile; TMT-B, Trail-Making test part B; and WMHs, white matter hyperintensities. levels were more strongly correlated with longitudinal MMSE scores in the MCI group than in controls.

\section{Discussion}

We present the first large study, to our knowledge, on plasma NFL in AD dementia. The main findings were that plasma NFL (1) correlated with CSF NFL independent of diagnosis, (2) was increased in the $\mathrm{AD}$ dementia group and in $\mathrm{A} \beta$-positive patients with MCI, (3) had diagnostic accuracy for AD dementia in the same range as established CSF biomarkers, and (4) was associated with cognitive deficits and neuroimaging hallmarks of $\mathrm{AD}$ at baseline and during follow-up. Together, these findings support that plasma NFL is a promising biomarker for neuronal injury in $\mathrm{AD}$, which may have potential for prognosis and monitoring of disease progression. This biomarker may be useful in clinical studies, in drug development, and ultimately in clinical practice. However, increased plasma NFLXc concentrations are also found in several other neurodegenerative disorders, such as progressive supranuclear palsy, frontotemporal dementia, and human immunodeficiency virus with brain engagement, ${ }^{11,12,15}$ meaning that it lacks disease specificity for AD. Therefore, we do not envision plasma NFL as a tool to differentiate AD from other neurodegenerative diseases. Rather, it may be valuable as a general biomarker for neurodegeneration.

Plasma NFL correlated with CSF NFL levels in the whole cohort and in diagnostic groups. Although these correlations were statistically significant, particularly in the whole cohort, the correlation coefficients were slightly lower than what was seen in previous studies of plasma or serum NFL levels, which included individuals with human immunodeficiency virus, ${ }^{11}$ progressive supranuclear palsy, ${ }^{12}$ other neurological diseases, ${ }^{10}$ and minor neurosurgical trauma. ${ }^{21}$ Hypothetically, it is possible that a greater variability in plasma NFL concentrations in AD compared with previously tested diseases could have influenced the correlations between plasma NFL and CSF NFL. Plasma NFL also correlated with other CSF biomarkers in the whole cohort, but those correlations were often not statistically significant within diagnostic groups, suggesting that they were confounded by diagnosis. This finding probably reflects that several different pathologic conditions are present in $\mathrm{AD}$ (eg, $\mathrm{A} \beta$ pathologic features, tau pathologic findings, and degeneration of different types of axons) and drive different biomarker responses, which will be weakly correlated overall. In MCI, a heterogeneous condition, high plasma NFL levles correlated with low CSF A 42 and high CSF t-tau, 
and this correlation supports the use of plasma NFL as a biomarker sensitive to $\mathrm{AD}$-related biological changes in prodromal AD. Plasma NFL also correlated with plasma tau in all diagnostic bagroups. Although plasma tau appears to be a weaker biomarker for neuronal injury than plasma NFL, ${ }^{22}$ these measures may partly reflect the same process (eg, axonal degeneration).

The AD dementia group had higher plasma NFL levels than the MCI group, and the MCI group and the AD dementia group had higher plasma NFL levels than the controls. Although plasma NFL overlaps between the diagnostic groups, the accuracy of plasma NFL for AD dementia vs controls is close to established CSF AD biomarkers and much higher than for plasma tau (AUROC, 0.87 vs 0.78 when adjusted for demographics and $A P O E$ \&4; AUROC, 0.79 vs 0.64 when adjusted only for demographics). To our knowledge, this study represents the first time that a peripheral, noninvasive biomarker for neuronal injury has shown diagnostic accuracy for $\mathrm{AD}$ dementia comparable with established biomarkers in a largescale multicenter cohort.

In the MCI group, plasma NFL levels were increased primarily in A $\beta$-positive patients with MCI (ie, prodromal AD). Plasma NFL concentration did not differ between clinically stable and progressive $\mathrm{A} \beta$-positive patients with MCI. This result may have been influenced by the short follow-up time used to define stable MCI (2 years), which may be too abbreviated to verify the benign nature of the so-called stable condition. ${ }^{23}$ The finding that plasma NFL concentrations were increased already in prodromal AD is promising because it may render plasma NFL more useful in drug development, which is largely focused on this early stage of $\mathrm{AD},{ }^{1}$ as well as in clinical practice because patients with MCI increasingly seek medical evaluation. Plasma NFL did not differ between $A \beta$-positive and $A \beta$ negative controls, suggesting that any neuronal injury that may have occurred in $\mathrm{A} \beta$-positive controls (ie, preclinical $\mathrm{AD}$ ) is below the detection limit for plasma NFL. This result is well in line with the theory that preclinical AD is devoid of substantial neuronal injury. ${ }^{24,25}$

The final major finding was that plasma NFL was associated with several cognitive and imaging AD hallmarks at baseline and when those measures were analyzed over time. Specifically, plasma NFL was associated with general cognition (MMSE score and ADAS-COG 11 score) and executive function (TMT-B score) at baseline and with decline in all tested cognitive measures over time. The somewhat stronger correlations with timed tests, including TMT-B and WAIS-R digit symbol substitution test, compared with the memory test may suggest that plasma NFL primarily reflects damage to larger myelinated axonal processes of neurons. For imaging measures, associations were seen with lateral ventricles, hippocampal volume, and AD cortex thickness at baseline and over time, as well as with hypometabolism over time. The correla- tions were strongest in the MCI group and the AD dementia group but were statistically significant in the whole cohort when adjusting for diagnosis.

\section{Limitations}

This study is limited by the lack of patients with neurodegenerative diseases other than $\mathrm{AD}$, which prevented our testing for disease specificity of plasma NFL. Another limitation is the restricted sample in the ADNI, such that patients with substantial vascular burden were excluded. This exclusion may have made it difficult to detect subtle associations between plasma NFL and white matter pathologic findings and may explain the surprising finding that plasma NFL did not correlate with WMHs, despite that CSF NFL has been considered a marker of white matter pathologic features, ${ }^{26}$ including in $\mathrm{AD} .^{7}$ Future studies should test plasma NFL in a more unselected group of patients with $\mathrm{AD}$ and may also explore different proxies for white matter injury. A larger age span should also be included in future work because it is possible that vascular comorbidities may change with age and alter the diagnostic accuracy of plasma NFL for patients with AD vs controls and other diseases.

All main results were in the expected directions, without illogical data. The main findings that plasma NFL concentration was increased in the $\mathrm{AD}$ dementia group and correlated with CSF NFL, imaging, and cognitive hallmarks of AD were all statistically significant. We believe that the consistency of these data makes it unlikely that they were falsely positive. Therefore, we reported $P$ values uncorrected for multiple comparisons.

\section{Conclusions}

We found that plasma NFL concentration is increased in $\mathrm{AD}$, even in prodromal disease, and that it correlates with important disease hallmarks, measured by cognitive tests, neuroimaging, and CSF biomarkers. The fact that plasma NFL concentration is also elevated in other neurological diseases ${ }^{11,12,15}$ and that NFL may be released from neurons in $\mathrm{A} \beta$-dependent and $\mathrm{A} \beta$-independent pathologic conditions $\mathrm{s}^{27} \mathrm{ar}$ gues against the use of plasma NFL for differential diagnosis of AD vs other dementias. However, plasma NFL may be a valuable noninvasive tool to assess neurodegeneration and to identify individuals at risk for future cognitive decline and brain atrophy. Therefore, plasma NFL is a promising peripheral biomarker for neurodegeneration, including in AD. In a clinical trial scenario, it is possible that plasma NFL may be used (together with demographics and $A P O E \varepsilon 4$ genotype data) to predict longitudinal disease progression. Future studies with repeated samples should test plasma NFL as a longitudinal noninvasive proxy for neurodegeneration.

\section{ARTICLE INFORMATION}

Accepted for Publication: December 16, 2016.

Published Online: March 27, 2017.

doi:10.1001/jamaneurol.2016.6117
Open Access: This is an open access article distributed under the terms of the CC-BY License. (c) 2017 Mattsson N et al. JAMA Neurology. Author Affiliations: Clinical Memory Research Unit, Department of Clinical Sciences, Faculty of Medicine, Lund University, Lund, Sweden
(Mattsson); Memory Clinic, Skåne University Hospital, Scania, Sweden (Mattsson); Department of Neurology, Skåne University Hospital, Scania, Sweden (Mattsson); Clinical Neurochemistry Laboratory, Sahlgrenska University Hospital, Mölndal, Sweden (Andreasson, Zetterberg, 
Blennow); Department of Psychiatry and Neurochemistry, Intitute of Neuroscience and Physiology, Sahlgrenska Academy at the University of Gothenburg, Möndal, Sweden (Andreasson, Zetterberg, Blennow); Department of Molecular Neuroscience, University College London Institute of Neurology, Queen Square, London, England (Zetterberg).

Author Contributions: Dr Mattsson had full access to all the data in the study and takes responsibility for the integrity of the data and the accuracy of the data analysis.

Study concept and design: Mattsson, Zetterberg, Blennow.

Acquisition, analysis, or interpretation of data: All authors.

Drafting of the manuscript: Mattsson.

Critical revision of the manuscript for important

intellectual content: Andreasson, Zetterberg,

Blennow.

Statistical analysis: Mattsson.

Obtained funding: Mattsson, Zetterberg, Blennow. Administrative, technical, or material support: Andreasson, Zetterberg, Blennow.

Conflict of Interest Disclosures: Dr Zetterberg reported serving on advisory boards for Roche Diagnostics, Pharmasum, and Eli Lilly and Company and reported being a Wallenberg Academy Fellow. Dr Blennow reported serving as a consultant to or on advisory boards for Alzheon, Eli Lilly and Company, Fujirebio Europe, IBL International, Novartis Pharmaceuticals Corporation, and Roche Diagnostics and reported holding a Torsten Söderberg Professorship in Medicine. Drs Zetterberg and Blennow reported being cofounders of Brain Biomarker Solutions in Gothenburg AB, a GU Ventures-based platform company at the University of Gothenburg. No other disclosures were reported.

Funding/Support: Data collection (and sharing) for this project was funded by the Alzheimer's Disease Neuroimaging Initiative (ADNI) (grant U01 AG024904 from the National Institutes of Health) and by the Department of Defense OD ADNI (award W81XWH-12-2-0012). The ADNI is funded by the National Institute on Aging and by the National Institute of Biomedical Imaging and Bioengineering, as well as through generous contributions from the following: AbbVie, Alzheimer's Association, Alzheimer's Drug Discovery Foundation, Araclon, Biotech, BioClinica Inc, Biogen, Bristol-Myers Squibb, CereSpir Inc, Cogstate, Eisai Inc, Elan Pharmaceuticals Inc, Eli Lilly and Company, Eurolmmun, F. Hoffmann-La Roche Ltd and its affiliated company Genentech Inc, Fujirebio Europe, GE Healthcare, IXICO Ltd, Janssen Alzheimer Immunotherapy Research \& Development LLC, Johnson \& Johnson Pharmaceutical Research \& Development LLC, Lumosity, Lundbeck, Merck \& Co Inc, Meso Scale Diagnostics LLC, NeuroRx Research, Neurotrack Technologies, Novartis Pharmaceuticals Corporation, Pfizer Inc, Piramal Imaging, Servier, Takeda Pharmaceutical Company, and Transition Therapeutics. The Canadian Institutes of Health Research provides funds to support ADNI clinical sites in Canada. Private-sector contributions are facilitated by the Foundation for the National Institutes of Health. The grantee organization is the Northern California Institute for Research and Education Inc, and the study is coordinated by the Alzheimer's Therapeutic Research Institute at the
University of Southern California. The ADNI data are disseminated by the Laboratory of Neuro Imaging at the University of Southern California. This research was also supported by grants from the Swedish Alzheimer Foundation, the Greta and Johan Kock Foundation, the Thelma Zoega Foundation, the Strategic Research Area MultiPark (Multidisciplinary Research in Parkinson's Disease) at Lund University, Vinnova, the Swedish Research Council, the European Research Council, Swedish State Support for Clinical Research, and Frimurarestiftelsen.

Role of the Funder/Sponsor: No sponsor had any role in the design and conduct of the study: collection, management, analysis, and interpretation of the data; preparation, review, or approval of the manuscript; and decision to submit the manuscript for publication. Data used in preparation of this article were obtained from the ADNI database. As such, the investigators within the ADNI contributed to the design and implementation of ADNI or provided data but did not participate in the analysis or writing of this report.

Group Information: Principal Investigator (PI): Michael W. Weiner, MD University of California San Francisco. Alzheimer's Disease Cooperative Study $\mathrm{PI}$ and Director of Coordinating Center Clinical Core: Paul Aisen, MD University of Southern California.

Executive Committee: UC San Francisco: Michael Weiner, MD; University of Southern California: Paul Aisen, MD; Arthur W. Toga, PhD; Mayo Clinic, Rochester, MN: Ronald Petersen, MD, PhD; Clifford R. Jack Jr MD; UC Berkeley: William Jagust, MD; University of Pennsylvania: John Q. Trojanowki, MD, PhD; Leslie M. Shaw; UC Davis: Laurel Beckett, PhD; Brigham and Women's Hospital/Harvard Medical School: Robert C. Green, MD; Indiana University: Andrew J. Saykin, PsyD; Washington University St. Louis: John Morris, MD. ADNI External Advisory Board (ESAB): Prevent Alzheimer's Disease 2020 Zaven Khachaturian, PhD (Chair); Siemens: Greg Sorensen, MD; Alzheimer's Association: Maria Carrillo, PhD; University of Pittsburgh: Lew Kuller, MD; Washington University St. Louis: Marc Raichle, MD; David Holtzman, MD; Cornell University: Steven Paul, MD; Albert Einstein College of Medicine of Yeshiva University: Peter Davies, MD; AD Drug Discovery Foundation: Howard Fillit, MD; Acumen Pharmaceuticals: Franz Hefti, PhD; Northwestern University:M. Marcel Mesulam, MD; National Institute of Mental Health: William Potter, MD Brown University: Peter Snyder.

ADNI 2 Private Partner Scientific Board (PPSB): Eli Lilly: Adam Schwartz, MD (Chair).

Data and Publications Committee: Brigham and Women's Hospital/Harvard Medical School: Robert C. Green, MD, MPH (Chair).

Resource Allocation Review Committee: University of Washington: Tom Montine, MD, PhD (Chair).

Clinical Core Leaders: Mayo Clinic, Rochester, MN Ronald Petersen, MD, PhD (Core PI); University of Southern California: Paul Aisen, MD.

Clinical Informatics and Operations: University of California San Diego: Ronald G. Thomas, PhD: Michael Donohue, PhD; Sarah Walter, MSc; Devon Gessert; Tamie Sather, MA; Gus Jiminez, MBS; Archana B. Balasubramanian, PhD; Jennifer Mason, $\mathrm{MPH}$; Iris Sim.
Biostatistics Core Leaders and Key Personnel: University of California Davis: Laurel Beckett, PhD (Core PI); Danielle Harvey, PhD; University of California San Diego: Michael Donohue, PhD.

MRI Core Leaders and Key Personnel: Mayo Clinic, Rochester, MN: Clifford R. Jack Jr (Core PI); Matthew Bernstein, PhD; Bret Borowski, RT; Jeff Gunter, PhD; Matt Senjem, MS; Prashanthi Vemuri, PhD; David Jones, MD; Kejal Kantarci; Chad Ward; University of London: Nick Fox, MD; UCLA School of Medicine: Paul Thompson, PhD; University of California San Francisco: Norbert Schuff, PhD; University of California Davis: Charles DeCarli, MD.

PET Core Leaders and Key Personnel: UC Berkeley: William Jagust, MD (Core PI); Susan Landau, PhD; University of Michigan: Robert A. Koeppe, PhD; University of Utah: Norm Foster, MD; Banner Alzheimer Institute: Eric M. Reiman, MD; Kewei Chen, PhD; University of Pittsburgh; Chet Mathis, MD.

Neuropathology Core Leaders:Washington University St. Louis: John C. Morris, MD; Nigel J. Cairns, PhD, FRCPath; Erin Franklin, MS, CCRP; Lisa Taylor-Reinwald, BA, HTL (ASCP) - Past Investigator.

Biomarkers Core Leaders and Key Personnel: University of Pennsylvania School of Medicine: Leslie M. Shaw, PhD; John Q. Trojanowki, MD, PhD; Virginia Lee, PhD, MBA; Magdalena Korecka, PhD; Michal Figurski, PhD.

Informatics Core Leaders and Key Personnel: University of Southern California: Arthur W. Toga, PhD (Core PI); Karen Crawford; Scott Neu, PhD.

Genetics Core Leaders and Key Personnel: Indiana University: Andrew J. Saykin, PsyD; Tatiana M. Foroud, PhD; Li Shen, PhD; Kelley Faber, MS, CCRC; Sungeun Kim, PhD; Kwangsik Nho, PhD; University of California Irvine: Steven Potkin, MD. Initial Concept Planning \& Development: University of California San Francisco: Michael W. Weiner, MD; University of California San Diego: Lean Thal, MD; Prevent Alzheimer's Disease 2020: Zaven Khachaturian, PhD (Chair).

Early Project Proposal Development: University of California San Diego: Leon Thal, MD; Neil Buckholtz National Institute on Aging; University of California San Francisco: Michael W. Weiner, MD; Brown University: Peter J. Snyder, PhD; National Institute of Mental Health: William Potter, MD; Cornell University: Steven Paul, MD; Johns Hopkins University: Marilyn Albert, PhD; Richard Frank Consulting: Richard Frank, MD, PhD; Prevent Alzheimer's Disease 2020: Zaven Khachaturian, $\mathrm{PhD}$ (Chair).

NIA: National Institute on Aging: John Hsiao, MD

Investigators by Site: Oregon Health \& Science University: Jeffrey Kaye, MD; Joseph Quinn, MD; Lisa Silbert, MD; Betty Lind, BS; Raina Carter, BA Past Investigator; Sara Dolen, BS - Past Investigator. University of Southern California: Lon S. Schneider, MD; Sonia Pawluczyk, MD; Mauricio Becerra, BS; Liberty Teodoro, RN; Bryan M. Spann, DO, PhD Past Investigator. University of California San Diego: James Brewer, MD, PhD; Helen Vanderswag, RN; Adam Fleisher, MD - Past Investigator. University of Michigan: Judith L. Heidebrink, MD, MS; Joanne L. Lord, LPN, BA, CCRC - Past Investigator. Mayo Clinic, Rochester, MN: Ronald Petersen, MD, PhD; Sara S. Mason, RN; Colleen S. Albers, RN; David 
Knopman, MD; Kris Johnson, RN - Past Investigator. Baylor College of Medicine: Rachelle S. Doody, MD, PhD; Javier Villanueva-Meyer, MD; Valory Pavlik, PhD; Victoria Shibley, MS; Munir Chowdhury, MBBS MS - Past Investigator; Susan Rountree, MD - Past Investigator; Mimi Dang, MD - Past Investigator. Columbia University Medical Center: Yaakov Stern, PhD; Lawrence S. Honig, MD, PhD; Karen L. Bell, MD. Washington University, St. Louis: Beau Ances, MD; John C. Morris, MD; Maria Carroll, RN, MSN; Mary L. Creech, RN, MSW; Erin Franklin, MS, CCRP; Mark A. Mintun, MD - Past Investigator; Stacy Schneider, APRN, BC, GNP - Past Investigator: Angela Oliver, RN, BSN, MSG - Past Investigator. University of Alabama Birmingham: Daniel Marson, JD, PhD; David Geldmacher, MD; Marissa Natelson Love, MD; Randall Griffith, PhD, ABPP - Past Investigator; David Clark, MD - Past Investigator; John Brockington, MD - Past Investigator; Erik Roberson, MD - Past Investigator. Mount Sinai School of Medicine: Hillel Grossman, MD; Effie Mitsis, PhD. Rush University Medical Center: Raj C. Shah, MD; Leyla deToledo-Morrell, PhD - Past Investigator. Wien Center: Ranjan Duara, MD; Mari T. Greig-Custo, MD; Warren Barker, MA, MS. Johns Hopkins University: Marilyn Albert, PhD; Chiadi Onyike, MD; Daniel D'Agostino II, BS; Stephanie Kielb, BS - Past Investigator. New York University: Martin Sadowski, MD, PhD; Mohammed O. Sheikh, MD; Anaztasia Ulysse; Mrunalini Gaikwad. Duke University Medical Center: P. Murali Doraiswamy, MBBS, FRCP; Jeffrey R. Petrella, MD; Salvador Borges-Neto, MD; Terence Z. Wong, MD - Past Investigator; Edward Coleman - Past Investigator. University of Pennsylvania: Steven E. Arnold, MD; Jason H. Karlawish, MD; David A. Wolk, MD; Christopher M. Clark, MD. University of Kentucky: Charles D. Smith, MD; Greg Jicha, MD; Peter Hardy PhD; Partha Sinha, PhD; Elizabeth Oates, MD; Gary Conrad, MD. University of Pittsburgh: Oscar L. Lopez, MD; MaryAnn Oakley, MA; Donna M. Simpson, CRNP, MPH. University of Rochester Medical Center: Anton P. Porsteinsson, MD; Bonnie S. Goldstein, MS, NP; Kim Martin, RN; Kelly M. Makino, BS - Past Investigator; M. Saleem Ismail MD - Past Investigator; Connie Brand, RN - Past Investigator. University of California Irvine: Steven G. Potkin, MD; Adrian Preda, MD; Dana Nguyen, PhD. University of Texas Southwestern Medical School: Kyle Womack, MD; Dana Mathews, MD, PhD; Mary Quiceno, MD. Emory University: Allan I. Levey, MD, PhD; James J. Lah, MD, PhD; Janet S. Cellar, DNP, PMHCNS-BC. University of Kansas Medical Center: Jeffrey M. Burns, MD; Russell H. Swerdlow, MD; William M. Brooks, PhD. University of California Los Angeles: Liana Apostolova, MD; Kathleen Tingus, PhD; Ellen Woo, PhD; Daniel H.S. Silverman, MD, PhD; Po H. Lu, PsyD - Past Investigator; George Bartzokis, MD - Past Investigator. Mayo Clinic Jacksonville: Neill R GraffRadford, MBBCH, FRCP (London); Francine Parfitt MSH, CCRC; Kim Poki-Walker, BA. Indiana University: Martin R. Farlow, MD; Ann Marie Hake, MD; Brandy R. Matthews, MD - Past Investigator; Jared R. Brosch, MD; Scott Herring, RN, CCRC. Yale University School of Medicine: Christopher $\mathrm{H}$. van Dyck, MD; Richard E. Carson, PhD; Martha G. MacAvoy, PhD; Pradeep Varma, MD. McGill University Montreal-Jewish General Hospital: Howard Chertkow, MD; Howard Bergman, MD; Chris Hosein, MEd. Sunnybrook Health Sciences, Ontario: Sandra Black, MD, FRCPC; Bojana Stefanovic, PhD; Curtis Caldwell, PhD. U.B.C. Clinic for AD \& Related Disorders: Ging-Yuek Robin Hsiung MD, MHSc, FRCPC; Benita Mudge, BS; Vesna Sossi, PhD; Howard Feldman, MD, FRCPC - Past Investigator; Michele Assaly, MA - Past Investigator. Cognitive Neurology St. Joseph's, Ontario: Elizabeth Finger, MD; Stephen Pasternack, MD, PhD; Irina Rachisky, MD; Dick Trost, PhD - Past Investigator; Andrew Kertesz, MD - Past Investigator. Cleveland Clinic Lou Ruvo Center for Brain Health: Charles Bernick, MD, MPH; Donna Munic, PhD. Northwestern University: Marek-Marsel Mesulam, MD; Emily Rogalski, PhD; Kristine Lipowski, MA; Sandra Weintraub, PhD; Borna Bonakdarpour, MD; Diana Kerwin, MD - Past Investigator; Chuang-Kuo Wu, MD, PhD - Past Investigator; Nancy Johnson, PhD - Past Investigator. Premiere Research Inst (Palm Beach Neurology): Carl Sadowsky, MD; Teresa Villena, MD. Georgetown University Medical Center: Raymond Scott Turner, MD, PhD; Kathleen Johnson, NP; Brigid Reynolds, NP. Brigham and Women's Hospital: Reisa A. Sperling, MD; Keith A. Johnson, MD; Gad Marshall, MD. Stanford University: Jerome Yesavage, MD; Joy L. Taylor, PhD; Barton Lane, MD; Allyson Rosen, PhD - Past Investigator; Jared Tinklenberg, MD - Past Investigator. Banner Sun Health Research Institute: Marwan N. Sabbagh, MD; Christine M. Belden, PsyD; Sandra A. Jacobson, MD; Sherye A. Sirrel, CCRC. Boston University: Neil Kowall, MD; Ronald Killiany, PhD; Andrew E. Budson, MD; Alexander Norbash, MD - Past Investigator; Patricia Lynn Johnson, BA - Past Investigator. Howard University Thomas O. Obisesan, MD, MPH; Saba Wolday, MSc; Joanne Allard, PhD. Case Western Reserve University: Alan Lerner, MD; Paula Ogrocki, PhD; Curtis Tatsuoka, PhD; Parianne Fatica, BA, CCRC. University of California, Davis - Sacramento: Evan Fletcher, PhD; Pauline Maillard, PhD; John Olichney, MD; Charles DeCarli, MD - Past Investigator; Owen Carmichael, PhD - Past Investigator. Neurological Care of CNY: Smita Kittur, MD - Past Investigator. Parkwood Hospital: Michael Borrie, MB ChB; T-Y Lee, PhD; Dr Rob Bartha, PhD. University of Wisconsin: Sterling Johnson, PhD; Sanjay Asthana, MD; Cynthia M. Carlsson, MD, MS. University of California, Irvine - BIC: Steven G. Potkin, MD; Adrian Preda, MD; Dana Nguyen, PhD. Banner Alzheimer's Institute: Pierre Tariot, MD; Anna Burke, MD; Ann Marie Milliken, NMD; Nadira Trncic, MD, PhD, CCRC - Past Investigator; Adam Fleisher, MD - Past Investigator; Stephanie Reeder, BA - Past Investigator. Dent Neurologic Institute: Vernice Bates, MD; Horacio Capote, MD; Michelle Rainka, PharmD, CCRP. Ohio State University: Douglas W. Scharre, MD; Maria Kataki, MD, PhD; Brendan Kelley, MD. Albany Medical College: Earl A. Zimmerman, MD; Dzintra Celmins, MD; Alice D. Brown, FNP. Hartford Hospital, Olin Neuropsychiatry Research Center: Godfrey D. Pearlson, MD; Karen Blank, MD; Karen Anderson, RN. Dartmouth-Hitchcock Medical Center: Laura A. Flashman, PhD; Marc Seltzer, MD; Mary L. Hynes, RN, MPH; Robert B. Santulli, MD - Past Investigator. Wake Forest University Health Sciences: Kaycee M. Sink, MD, MAS; Leslie Gordineer; Jeff D. Williamson, MD, MHS - Past Investigator; Pradeep Garg, PhD Past Investigator; Franklin Watkins, MD - Past Investigator. Rhode Island Hospital: Brian R. Ott, MD; Geoffrey Tremont, PhD; Lori A. Daiello, Pharm.D, ScM. Butler Hospital: Stephen Salloway, MD, MS; Paul Malloy, PhD; Stephen Correia, PhD. UC San Francisco: Howard J. Rosen, MD; Bruce L. Miller, MD; David Perry, MD. Medical University
South Carolina: Jacobo Mintzer, MD, MBA; Kenneth Spicer, MD, PhD; David Bachman, MD. St. Joseph's Health Care: Elizabeth Finger, MD; Stephen Pasternak, MD; Irina Rachinsky, MD; John Rogers, MD; Andrew Kertesz, MD - Past Investigator; Dick Drost, MD - Past Investigator. Nathan Kline Institute: Nunzio Pomara, MD; Raymundo Hernando, MD; Antero Sarrael, MD. University of lowa College of Medicine: Susan K. Schultz, MD; Karen Ekstam Smith, RN; Hristina Koleva, MD; Ki Won Nam, MD; Hyungsub Shim, MD- Past Investigator. Cornell University: Norman Relkin, MD, PhD; Gloria Chiang, MD; Michael Lin, MD; Lisa Ravdin, PhD. University of South Florida: USF Health Byrd Alzheimer's Institute: Amanda Smith, MD; Balebail Ashok Raj, MD; Kristin Fargher, MD- Past Investigator.

\section{REFERENCES}

1. Mattsson N, Carrillo MC, Dean RA, et al. Revolutionizing Alzheimer's disease and clinical trials through biomarkers. Alzheimers Dement (Amst). 2015;1(4):412-419.

2. Blennow $\mathrm{K}$, Hampel $\mathrm{H}$, Weiner $\mathrm{M}$, Zetterberg $\mathrm{H}$. Cerebrospinal fluid and plasma biomarkers in Alzheimer disease. Nat Rev Neurol. 2010;6(3):131144

3. Frisoni GB, Fox NC, Jack CR Jr, Scheltens $P$, Thompson PM. The clinical use of structural MRI in Alzheimer disease. Nat Rev Neurol. 2010;6(2):67-77.

4. Nordberg A, Rinne JO, Kadir A, Långström B. The use of PET in Alzheimer disease. Nat Rev Neurol. 2010;6(2):78-87.

5. Blennow K, Mattsson N, Schöll M, Hansson O, Zetterberg H. Amyloid biomarkers in Alzheimer's disease. Trends Pharmacol Sci. 2015;36(5):297-309.

6. Zetterberg H. Neurofilament light: a dynamic cross-disease fluid biomarker for neurodegeneration. Neuron. 2016;91(1):1-3.

7. Zetterberg H, Skillbäck T, Mattsson $N$, et al: Alzheimer's Disease Neuroimaging Initiative. Association of cerebrospinal fluid neurofilament light concentration with Alzheimer disease progression. JAMA Neurol. 2016;73(1):60-67.

8. Gaiottino J, Norgren N, Dobson R, et al. Increased neurofilament light chain blood levels in neurodegenerative neurological diseases. PLoS One. 2013;8(9):e75091.

9. Bacioglu M, Maia LF, Preische O, et al. Neurofilament light chain in blood and CSF as marker of disease progression in mouse models and in neurodegenerative diseases. Neuron. 2016;91(1): 56-66.

10. Kuhle J, Barro C, Andreasson U, et al. Comparison of three analytical platforms for quantification of the neurofilament light chain in blood samples: ELISA, electrochemiluminescence immunoassay and Simoa. Clin Chem Lab Med. 2016; 54(10):1655-1661.

11. Gisslén M, Price RW, Andreasson U, et al. Plasma concentration of the neurofilament light protein (NFL) is a biomarker of CNS injury in HIV infection: a cross-sectional study. EBioMedicine. 2015;3:135-140.

12. Rojas JC, Karydas A, Bang J, et al. Plasma neurofilament light chain predicts progression in progressive supranuclear palsy. Ann Clin Transl Neurol. 2016;3(3):216-225

13. Petersen RC, Aisen PS, Beckett LA, et al. Alzheimer's Disease Neuroimaging Initiative 
(ADNI): clinical characterization. Neurology. 2010; 74(3):201-209.

14. McKhann G, Drachman D, Folstein M, Katzman $R$, Price $D$, Stadlan EM. Clinical diagnosis of Alzheimer's disease: report of the NINCDS-ADRDA Work Group under the auspices of Department of Health and Human Services Task Force on Alzheimer's Disease. Neurology. 1984;34(7):939-944.

15. Rohrer JD, Woollacott IOC, Dick KM, et al. Serum neurofilament light chain protein is a measure of disease intensity in frontotemporal dementia. Neurology. 2016;87(13):1329-1336.

16. Shaw LM, Vanderstichele $H$, Knapik-Czajka M, et al; Alzheimer's Disease Neuroimaging Initiative. Cerebrospinal fluid biomarker signature in Alzheimer's Disease Neuroimaging Initiative subjects. Ann Neurol. 2009;65(4):403-413.

17. Jack CR Jr, Bernstein MA, Fox NC, et al. The Alzheimer's Disease Neuroimaging Initiative (ADNI): MRI methods. J Magn Reson Imaging. 2008;27(4):685-691.
18. Jack CR Jr, Wiste HJ, Weigand SD, et al. Different definitions of neurodegeneration produce similar amyloid/neurodegeneration biomarker group findings. Brain. 2015;138(pt 12):3747-3759.

19. Schwarz C, Fletcher E, DeCarli C, Carmichael O. Fully-automated white matter hyperintensity detection with anatomical prior knowledge and without FLAIR. Inf Process Med Imaging. 2009;21:239-251.

20. Landau SM, Mintun MA, Joshi AD, et al; Alzheimer's Disease Neuroimaging Initiative. Amyloid deposition, hypometabolism, and longitudinal cognitive decline. Ann Neurol. 2012;72 (4):578-586.

21. Bergman J, Dring A, Zetterberg $\mathrm{H}$, et al. Neurofilament light in CSF and serum is a sensitive marker for axonal white matter injury in MS. Neurol Neuroimmunol Neuroinflamm. 2016;3(5):e271.

22. Mattsson $\mathrm{N}$, Zetterberg $\mathrm{H}$, Janelidze $\mathrm{S}$, et al; ADNI Investigators. Plasma tau in Alzheime disease. Neurology. 2016;87(17):1827-1835.
23. Buchhave $P$, Minthon $L$, Zetterberg $H$, Wallin AK, Blennow K, Hansson O. Cerebrospinal fluid levels of $\beta$-amyloid 1-42, but not of tau, are fully changed already 5 to 10 years before the onset of Alzheimer dementia. Arch Gen Psychiatry. 2012;69 (1):98-106.

24. Jack CR Jr, Knopman DS, Jagust WJ, et al. Hypothetical model of dynamic biomarkers of the Alzheimer's pathological cascade. Lancet Neurol. 2010;9(1):119-128.

25. Vos SJ, Xiong C, Visser PJ, et al. Preclinical Alzheimer's disease and its outcome: a longitudinal cohort study. Lancet Neurol. 2013;12(10):957-965.

26. Mattsson N. CSF biomarkers in neurodegenerative diseases. Clin Chem Lab Med. 2011;49(3):345-352

27. Mattsson N, Insel PS, Palmqvist $S$, et al; Alzheimer's Disease Neuroimaging Initiative. Cerebrospinal fluid tau, neurogranin, and neurofilament light in Alzheimer's disease. EMBO Mol Med. 2016;8(10):1184-1196. 